\title{
Soft Tissue Augmentation as a Method of Reducing the Liability to Pressure Sores in Spinal Injured Patients
}

\author{
R. A. Sutton, F.R.C.S., Ed., ${ }^{1}$ R. J. Minns, B.Eng., M.Sc., Ph.D., ${ }^{2}$ A. J. \\ Sherrin, B.Sc., ${ }^{3}$ J. Brown, B.Sc., Ph.D., ${ }^{4}$ J. C. Reignier, ${ }^{5}$ and M. Herlant. ${ }^{6}$ \\ ${ }^{1}$ Consultant Orthopaedic Surgeon, North of England Regional Spinal Injuries Unit, \\ Hexham General Hospital, Hexham, Northumberland, England, ${ }^{2}$ Senior Physicist, \\ Department of Medical Physics, Dryburn Hospital, Dryburn, Durham, England, \\ ${ }^{3}$ Product Manager, 'Medicarb', Dunlop Medical Products, Coventry, England, \\ ${ }^{4}$ Senior Biomedical Engineer, Dunlop Medical Products, Coventry, England, ${ }^{5}$ Con- \\ sultant Orthopaedic Surgeon, Centre de l'Arche, Etablissement Médico-Chirugical \\ et Ortho-Prothétique, St Saturnin, 72650 La Milesse, Le Mans, France, ${ }^{6}$ Medicin \\ Chef d'Etablissement, Directeur Médico-Technique, Centre de Rééducation Fonc- \\ tionelle, 25 Pavé du Moulin, 59260 Lille, Hellennes, France.
}

\section{Summary}

The development of a procedure designed to reduce the liability to pressure sores over bony prominences is described. This employs the properties of a particular type of carbon fibre pad implanted surgically over the bony prominence. The mechanical response of the pad to loading has been examined. The host tissue response to the pad involves penetration of the interstices between the carbon fibres with organised fibrous tissue. The host-tissue-carbon fibre pad complex has been found to be highly vascular. An 'ischiobarograph' has been developed and used in order to evaluate the alteration in pressure profile produced by the presence of the implanted prosthesis (the patient being in a sitting position).

Key words: Pressure sores; Prophylaxis; Carbon fibre.

\section{Introduction}

It has been estimated that about $68^{\circ}{ }_{0}$ of patients with spinal cord injury develop pressure sores at one time or another (Kosiak, 1959). A survey of all new cases of traumatic spinal paralysis treated in our Unit from 1975-1979 revealed that $33^{\circ}$. o had developed one or more sores during that period (Sutton et al., 1982). These figures were reflected in the workload of the Unit $-20^{\circ}{ }_{0}$ of the beds at that time being occupied by patients requiring surgical treatment for their pressure sores. This percentage is now falling.

Factors which predispose spinal cord injured patients to pressure sores include the following:

1. Presence of weightbearing bony prominence, for example ischial tuberosity (one of Guttmann's intrinsic factors; Guttmann, 1973).

2. External pressure-for example body weight acting on ischial tuberosities in sitting position (Guttmann's extrinsic factor). When loaded, soft tissues 
around bony prominences may be subjected to compression which, in time, may restrict tissue perfusion. Pressure sores are debilitating and, if not controlled, may give rise to serious and even fatal complications. Whether treated surgically (by direct closure or skin flap rotation) or conservatively, the soft tissue covering bony prominences is frequently disappointingly thin.

Bearing in mind these aetiological factors, a technique has been developed which shows clinical promise of a reduction in the development of pressure sores.

\section{Rationale of carbon fibre technique}

Our initial interest has been in ischial sores. The relative prominence of the ischium can be reduced by trimming. If the load-bearing area of the ischium (or remnant) could be altered to make it broader and less angular, the pressure in this area upon weightbearing would be further reduced. The 'tissue-shearing' effect at this site may also be further reduced. It was felt that the properties of carbon fibre could be employed in order to achieve this effect. Carbon is a material that is now known to be histologically acceptable (Tayton, 1982) and carbon in fibre form has been used in the body for replacement of tendon (Jenkins et al., 1977). In the present application, carbon fibre is not used in tension, thus avoiding the problems associated with this, as encountered in tendon replacement.

Carbon fibre is made by carbonising polyacrylonitrile fibres under carefully controlled conditions to produce a highly structured strong fibre of about 9 microns in diameter. Fibres can be converted into fabric form using specially adapted textile technology. It was therefore possible to produce a cushion-like structure made from a highly bioacceptable material which would achieve the effect referred to above (Fig. 1). A joint project was undertaken with Dunlop who already possessed a considerable expertise in carbon fibre for aerospace applications. A special fabric structure of various thicknesses $(4,9,15$, and

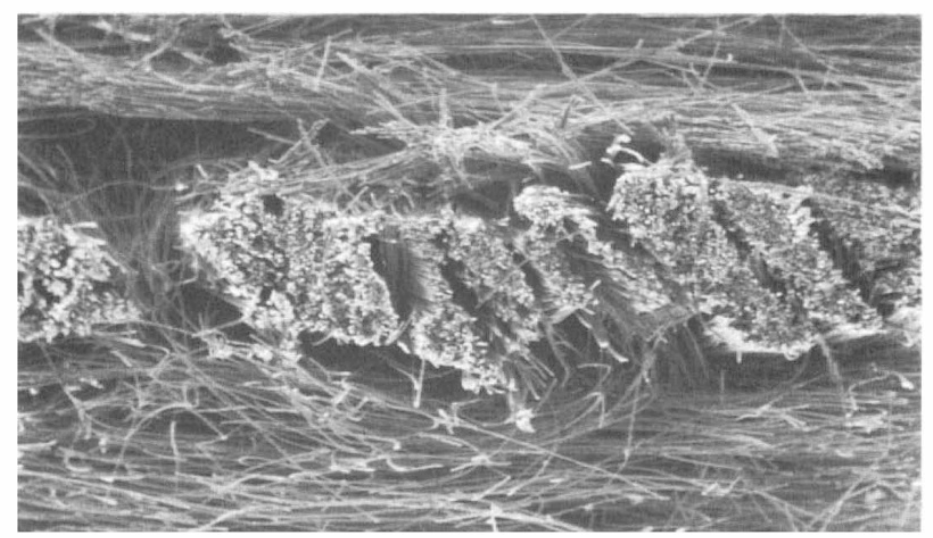

Figure 1 Scanning electron micrograph of a transverse section of carbon fibre pad showing arrangement of the carbon fibre bundles. 
$25 \mathrm{~mm}$ ) was designed to combine compliance with stability and compression resistance. The material is easily sterilised by normal procedures and can be worked with conventional theatre instruments.

\section{Assessment of results}

1. In order to provide a measure of the reduction of pressure achieved under bony prominences, a modification of the pedobarograph has been developed by one of the authors (R.J.M.). This will not be described in full here as a description of the equipment appears elsewhere (Minns et al., 1984).

In effect, a representation of the pressure distribution at the skin-glass interface is displayed on a television monitor screen as a coloured contour diagram. Increasing values of pressure are represented by different colours on the monitor so that it is easy to visualise the overall pattern of pressure distribution. In practice the patient would be sitting on some form of wheelchair cushion which would tend to reduce the pressure generated under the ischial tuberosities. Nevertheless it is felt that a comparison of the pressure patterns obtained preoperatively and postoperatively using the ischiobarograph is a very reliable method of assessing the effect of surgery in this respect. The system is therefore much more discerning than placing reliance on the length of time taken before another sore develops.

Note: Equipment for pressure monitoring at the Centre de l'Arche presents an equivalent picture.

2. The overall clinical progress of the patient was monitored regularly, including direct clinical examination of the area treated surgically.

\section{Summary of preclinical animal experiments}

Confirmation was required that host tissue would infiltrate the interstices of the carbon fibre pad when the pad was inserted under the deep fascia. Ease of subsequent removal and the possibility of fragmentaton of the pad also required evaluation.

Twenty hooded Wista rats were used in these experiments. Bilateral skin flaps were dissected on the dorsal thoracic area of the rats and carbon fibre pads of $1 \mathrm{~cm}$ diameter and $3 \mathrm{~mm}$ thick were implanted under the deep fascia on one side only. All the skin flaps were closed and sutured (the rats were unable to reach these wounds). Implants were retrieved at intervals of one, $2,4,8,16$, and 28 weeks; removal presented no difficulty. The specimens were examined using a variety of techniques.

First, the mechanical properties of the specimens were examined. Physical tests were conducted on the specimens using an Instron type 1195 universal testing machine. Compression tests were conducted on skin (20), carbon fibre pads (20), and the implanted material at a constant displacement rate of $5 \mathrm{~mm} / \mathrm{min}$. up to a stress of $6 \mathrm{MPa}$. The pads were then allowed to relax at a constant displacement for 10 minutes.

The stress-strain curves (Fig. 2) were plotted using nominal stress values from an initial diameter of $1 \mathrm{~cm}$; the strain was calculated from crosshead dis- 
placement and the specimens original thickness. The stiffness was calculated from the slope of the stress-strain curves at $6 \mathrm{MPa}$ stress, and the energy absorbed by the specimen was measured from the area under the curves, both values were plotted against implantation time. The load after 10 minutes relaxation was used to give a measure of the time dependent properties.

The stress-strain behaviour of the pad (and contained host tissue) very closely matched that of skin by 8 weeks (see Fig. 2).

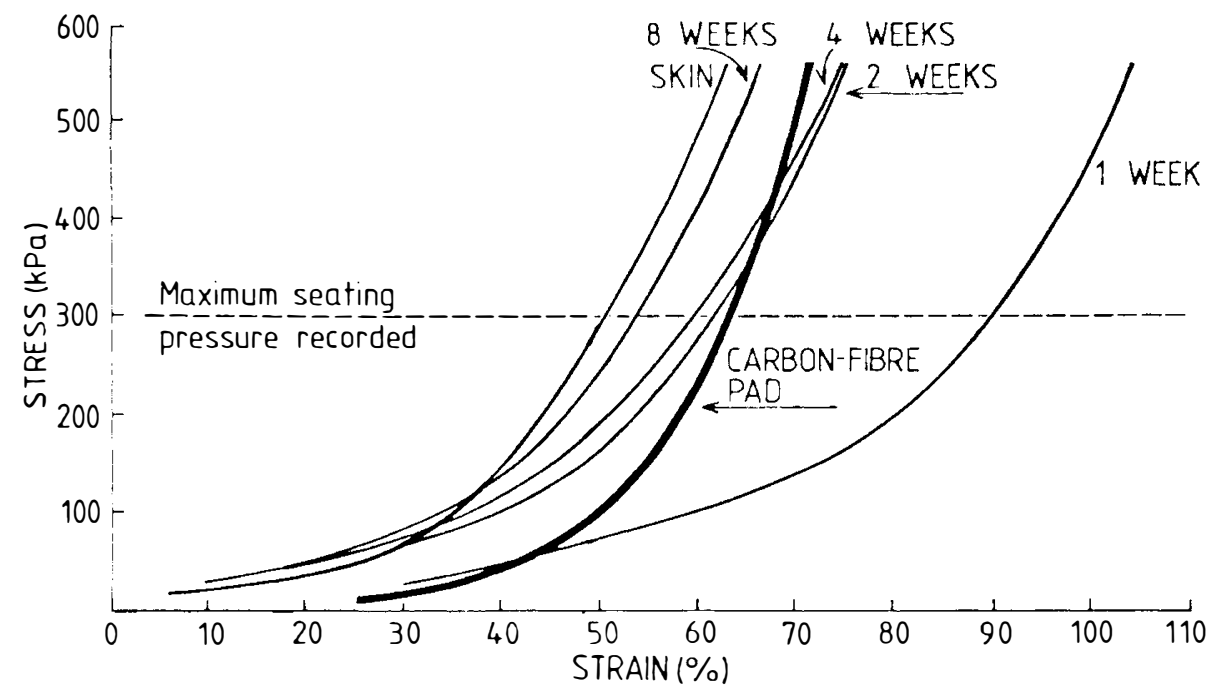

Figure 2 Stess-strain curves of the implanted pads tested in compression.

Secondly, histology was performed on fixed specimens. Thick sections (10 microns) were obtained from both the implanted and control tissue and overlying skin. These were stained with Van Geison and Weigarts stain. Histologically there appeared to be very little inflammatory response and there were only a few, randomly dispersed, giant multinuclear cells present. The predominant effect was a healthy fibroblastic appearance with large bundles of collagen fibres intertwined and following the architecture formed by the carbon fibres within the implant (Fig. 3). No carbon was found in the para-aortic or regional lymph nodes.

Thirdly, scanning electron microscopy was performed on sections through the implant. For this the specimens were dried using acetone and carbon dioxide in a critical point drying apparatus, vacuum coated in gold/palladium, and observed in a Cambridge S600 stereo-scan electron microscope operating at an accelerating voltage of $15 \mathrm{Kv}$.

Scanning electron microscopy of the pad at 8 weeks displayed the layered structure of the carbon fibre with no evidence of fragmentation or disruption and new fibrous tissue appeared well integrated into the pad (Fig. 4).

\section{Patient selection}

The procedure was in the first instance expected to be of value to the well adjusted and co-operative patient who has suffered from repeated sores in rela- 


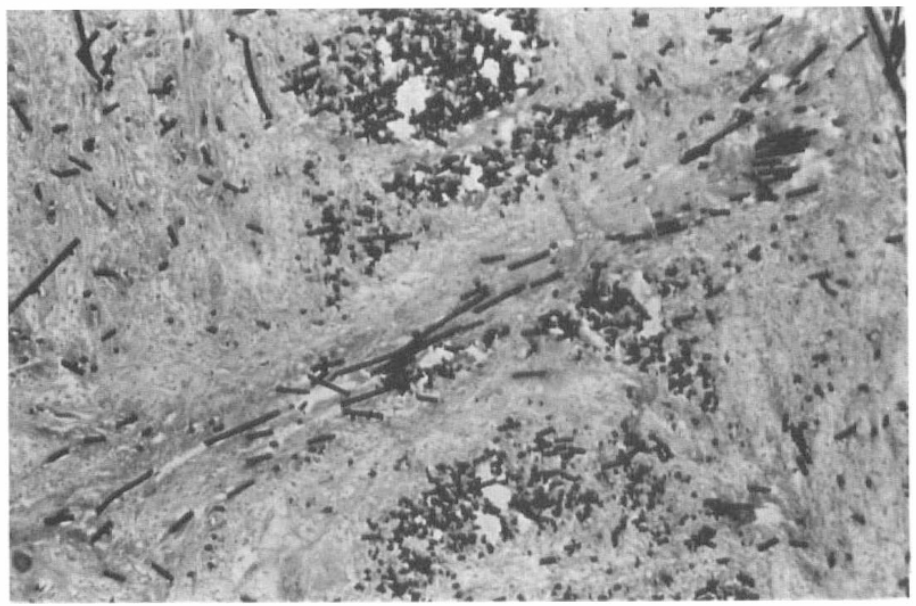

Figure 3 Transverse section of a carbon fibre pad at 8 weeks in the rat showing fibroblastic tissue response (carbon fibres at 6-9 micrometres in diameter).

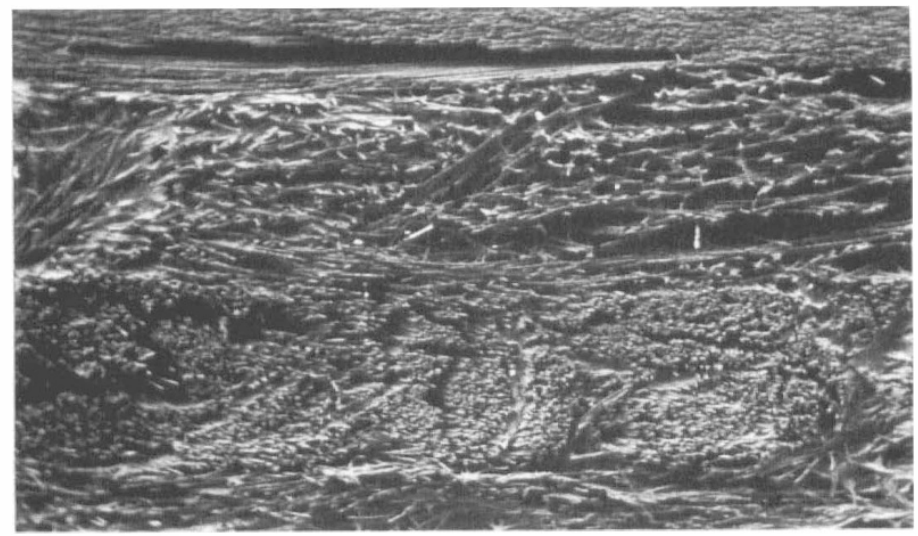

Figure 4 Scanning electron microscopy of a carbon fibre pad 8 weeks after implantation into rat.

tion to the underlying ischial tuberosity. This type of patient will have repeatedly spent long periods in bed in order to achieve healing of the sore. Despite this, recurrent breakdown of the area will have occurred when normal activity has been resumed.

Initial concern was with ischial sores. However, the technique is equally applicable to other sores overlying bony prominences and we have extended the technique to include some of these.

As confidence has been gained in the system, some patients have been included who have repeatedly displayed signs of an impending sore and who have had to spend considerable periods either in bed or avoiding particular positions. In one instance, the technique has been used as a method of soft tissue augmentation partly for 'mechanical' and partly for cosmetic reasons (patient M.A.F.). 

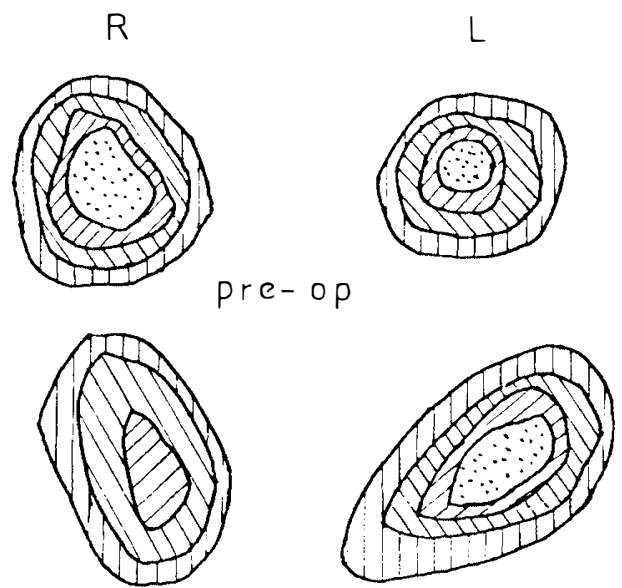

post op R
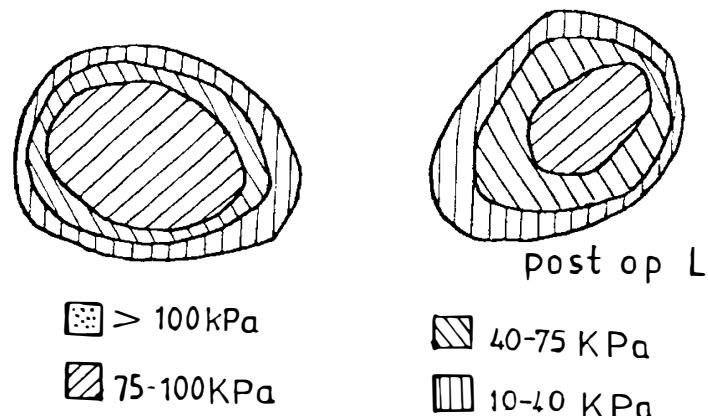

$\triangle 10-75 \mathrm{KPa}$

W10-40 KPa

Figure 5 Ischiobarograph recordings showing reduction in loading under each ischium postoperatively.

\section{Principles of operative technique}

It is not proposed to discuss the details of the operative technique in this paper. The principles of the procedure are as follows:

1. If there has been a previous pressure sore it should be healed prior to the insertion of the C-F pad.

2. The position of the previous sore should be marked out on the skin and in the case of ischial sores, the hips should be flexed to $90^{\circ}$ while this marking is carried out (in order to ensure that the position of the C-F pad adequately overlies the ischium when the patient is in the sitting position).

3. The incision associated with the insertion of the C-F pad should not overlie the area involved by the pressure sore.

4. The skin flap should be full thickness down to the deep fascia.

5. Underlying bone should be carefully trimmed and remodelled.

6. Haemostasis should be secured.

7. A pattern should be used to enable the C-F pad to be adequately shaped prior to the insertion. 

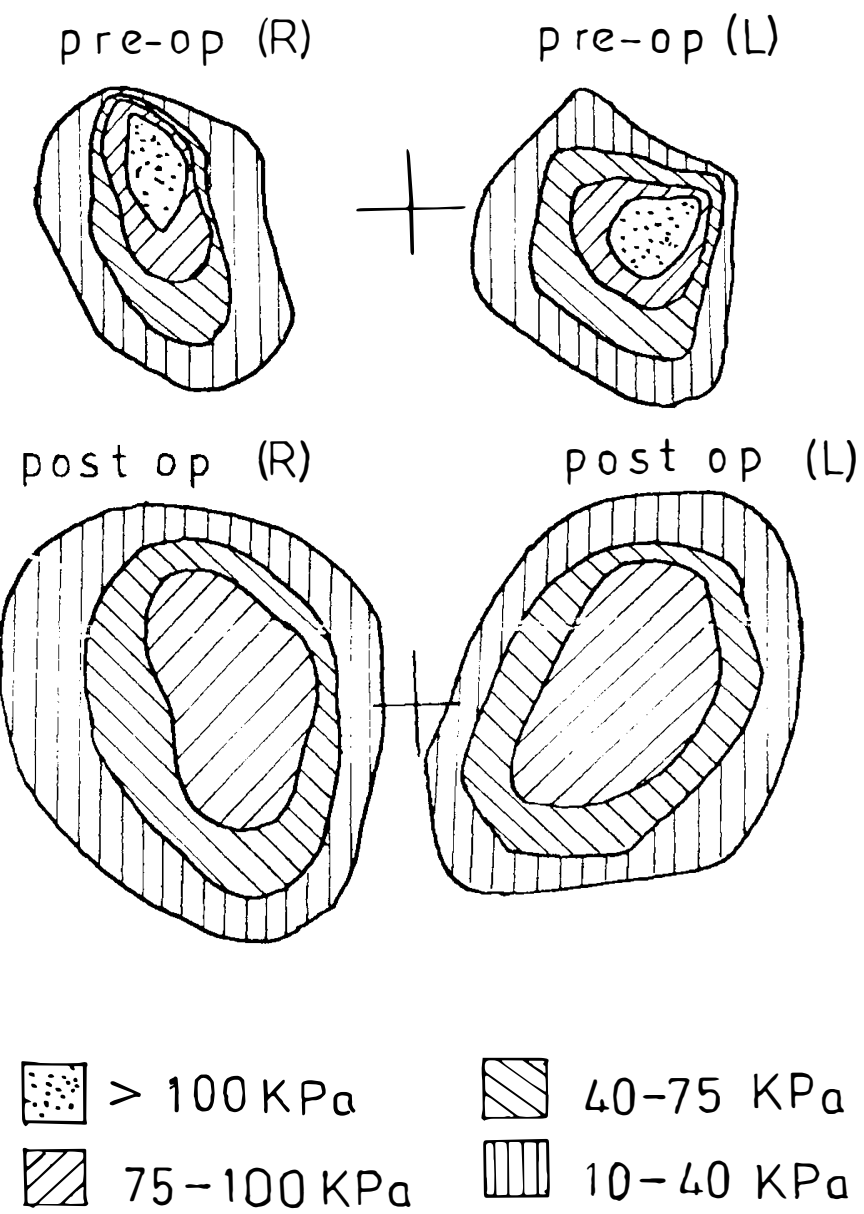

Figure 6 Ischiobarograph recordings showing areas where pressure bands are close together (high 'sheer') preoperatively. Wider separation is shown postoperatively as well as elimination of highest pressure band. The cross marks centre of mass.

8. The C-F pad should be positioned to cover the underlying bone and sutured into position.

9. Readyvac drainage should be established reliably and evacuation tubes within the operative site should be positioned to allow optimal drainage. (A topical antibiotic spray was used in all cases treated in England.)

10. Postoperatively a period of 6 weeks in bed was advised and the patient was nursed in a manner which avoided pressure being applied to the pads during this time. (During this time, adequate infiltration of the pad by host tissue should have occurred.)

\section{Ischiobarograph recordings}

The ischial sores have been evaluated on the ischiobarograph. Sores at other sites do not easily lend themselves to evaluation by this method. All ischiobarograph recordings display a reduction in loading under the ischial tuberosity 
Table I North of England Regional Spinal Injuries Unit

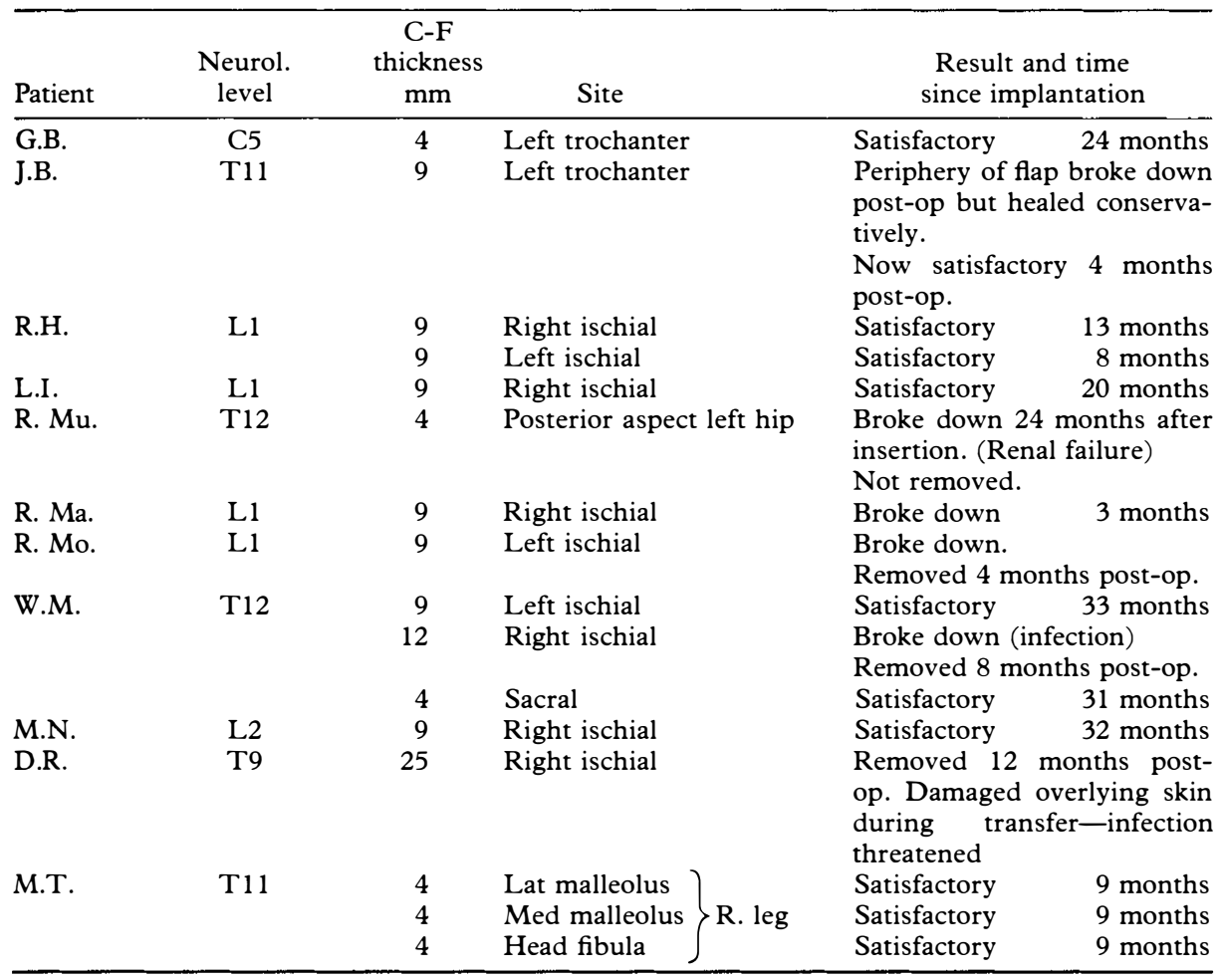

Table II Centre de l'arche

\begin{tabular}{|c|c|c|c|c|}
\hline Patient & $\begin{array}{c}\text { Neurol. } \\
\text { level }\end{array}$ & $\begin{array}{c}\mathrm{C}-\mathrm{F} \\
\text { thickness } \\
\mathrm{mm}\end{array}$ & Site & $\begin{array}{l}\text { Result and time } \\
\text { since implantation }\end{array}$ \\
\hline M.A. & $\mathrm{T} 7$ & 9 & Left ischial & Broke down post-op. \\
\hline M.A.F & & 9 & $\begin{array}{l}\text { Sternum soft tissue aug- } \\
\text { mentation }\end{array}$ & Satisfactory \\
\hline P.L. & $\mathrm{T} 7$ & 9 & Right ischial & $\begin{array}{l}\text { Broke down } 10 \text { months post- } \\
\text { op. }\end{array}$ \\
\hline J.P. & & $\begin{array}{l}9 \\
9 \\
4 \\
4\end{array}$ & $\left.\begin{array}{l}\text { Right ischial } \\
\text { Left ischial } \\
\text { Right trochanter } \\
\text { Left trochanter }\end{array}\right\}$ & Satisfactory \\
\hline
\end{tabular}

concerned postoperatively. An example of this effect is shown in Fig. 5 where the procedure was initially carried out at the right ischium and later at the left (Case R.H.). In cases where the initial ischiobarograph readings have shown areas where the pressure levels are close together, (implying a high tissue-shear effect), the postoperative record has shown a wider separation (Fig. 6).

\section{Blood pool scan}

A localised increase in skin temperature is discernible over the site of the C-F pads. A blood-pool was therefore carried out in one case (D.R.). $2 \mathrm{ml}$ of 
'Amerscan' stannous blood labelling agent was injected i.v. 30 minutes later a solution containing $500 \mathrm{MBq}$ of Tc $99 \mathrm{~m}$ eluate was given i.v. A general electric Maxicanna $400 \mathrm{~T}$ gamma camera was used for imaging. Paramount records were made of resulting scans using a multi-image formatter of the trochanteric or ischial regions.

The scan, carried out at 8 months after insertion of the pad, confirmed the presence of a prominent degree of vascularisation (Fig. 7).

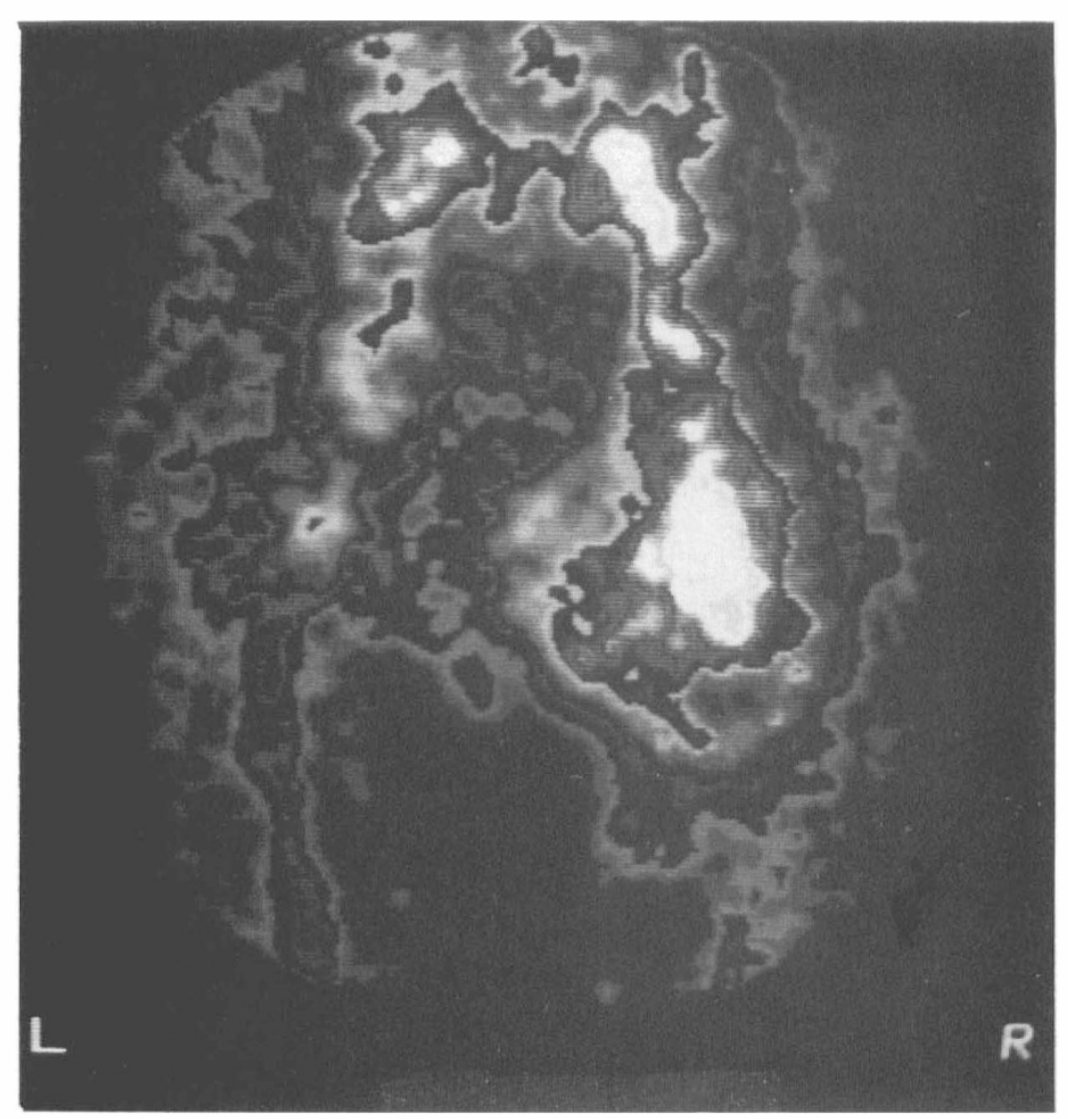

Figure 7 Blood pool scan showing high vascularity in the right ischial area (the site of the implanted carbon fibre pad). White areas correspond with maximum vascularity.

\section{Thermography}

Facilities for liquid crystal contact thermography have been obtained. Examination of patient G.B. 21 months after insertion of C-F pad $(4 \mathrm{~mm})$ over the left trochanter (a) recorded a persisting elevation in overlying temperature (Fig. 8). Details of the method of thermographic evaluation are provided in Appendix I.

\section{Discussion}

In all, $23 \mathrm{C}-\mathrm{F}$ pads have been inserted with an average follow-up of 14.8 months 

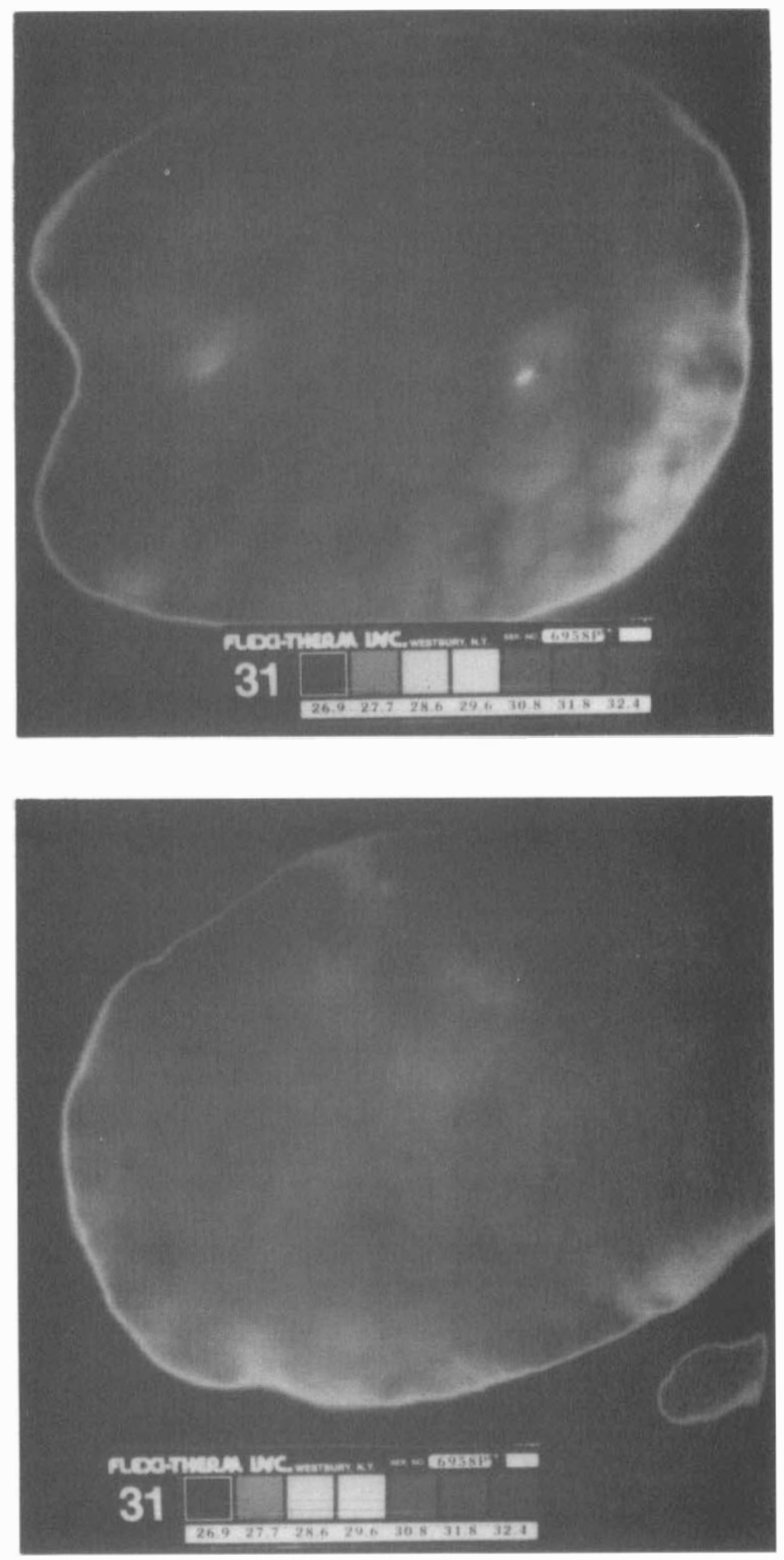

Figure 8 Thermograms of the trochanteric area 21 months after C-F pad insertion. a. implant side, b. controlateral side, without implant. 
postoperatively. (One patient has been followed up for only 4 months. Apart from this, a minimum follow-up period of 8 months has occurred and a maximum of 2 years 9 months.) Of these 23 pads inserted, seven sites have unfortunately broken down. Of the $15 \mathrm{C}-\mathrm{F}$ pads inserted into areas where there had previously been an open sore, five have been removed subsequently and another may have to be removed. One of these (the first pad inserted) proved to be too thick and become unduly prominent when connective tissue infiltration had occurred. This patient damaged the overlying skin during a transfer and the pad was therefore removed. The remaining pads were removed because of infection. Removal of a pad presents no undue difficulty. No persisting sinuses have been left though two cases were slow to heal.

Eight pads were inserted 'prophylactically'. At these sites, the skin had threatened to break down repeatedly. In the case of 'sitting' sores, the patients had been forced to rest in bed repeatedly in order to avoid breakdown. In the case of 'lying' sores, patients had not been able to lie safely on one side or the other without risk of skin breakdown.

The skin over one of these eight pads broke down. This occurred 2 years after insertion, at a time when the patient had developed marked uraemia due to end stage renal failure caused by amyloidosis. By this time the patient was no longer able to care for himself and the skin broke down when he was in a renal unit. The patient was subsequently nursed in the Spinal Injury Unit and the skin over the C-F pad at one stage had virtually healed over the pad. Recurrent breakdown occurred however when the patient's general condition deteriorated again terminally.

Sixteen $\left(70^{\circ}{ }_{0}\right)$ of the C-F pads are 'functioning' satisfactorily. Clinically the areas are well rounded in appearance and a reasonable thickness of tissue is palpable over the prominence involved. The borders of the pads are impalpable. These patients are now able to spend considerably longer periods lying or sitting on the areas concerned without harmful effects becoming apparent. In one case, for example, the patient was forced to wait 20 hours in an airport and his ischial areas (bilateral $9 \mathrm{~mm} \mathrm{C-F} \mathrm{pads)} \mathrm{survived} \mathrm{intact.}$

Experience has revealed that patient selection is important. The most suitable patient is one who looks after himself well (or is cared for well) but yet threatens to develop (or actually develops) a sore. Only a thin covering of soft tissue is present over bony prominences. In these circumstances this procedure can be expected to allow the patient to considerably extend the time spent in his wheelchair out of bed, or to lie on an area previously at risk when in bed.

\section{Conclusion}

The long-term management of pressure sores in this group of patients is notoriously difficult. The overall rate of successful implantations $(74 \%)$ is considered encouraging since this is associated with a considerable extension of the patients' freedom to return to an active wheelchair life. This percentage of successful implantations could be expected to improve further as the result of experience now gained.

How long into the future these advantages will extend remains to be seen. 
The situation to date encourages a continuing cautious development of the technique.

\section{Summary}

The development of pressure sores is a common complication of paraplegia. A surgical technique employing specially designed C-F pads has been described. The C-F pads represent a method of achieving tissue augmentation. This technique has been used in conjunction with usual methods of treatment of sores. The pads are inserted only after satisfactory skin cover has been achieved. In our initial trial cases, evaluation using an 'ischiobarograph' suggests that a reduction in pressure has been achieved in respect of the ischial sores evaluated. We believe that results in our first few cases have been sufficiently encouraging to continue cautious development of the technique.

\section{Acknowledgements}

The authors would like to express their sincere appreciation to the following people: Dr J. Sunter, Dr G. B. Singh, Miss J. E. Donkin and Mr G. P. Swinton for the histology. Professor G.R. Higginson for facilities for mechanical testing. Professor M. S. Morgan for the animal facilities. Professor K. Boddy and Dr P. J. Lowe at Regional Medical Physics Department at Newcastle General Hospital for advice with the project. Also Mrs J. McKee for inexhaustible typing.

\section{Appendix}

\section{Thermographic studies}

A Flexi-therm * Mk II contact thermography system was used in which a liquid crystal membrane forms one side of an inflatable box $28 \mathrm{~cm} \times 36 \mathrm{~cm}$. A transparent viewing window forms the other side of the box and when the detector is brought in contact with the area being examined, the flexibility of the membrane allows a high degree of conformability to the anatomy (Pochaczevsky et al., 1982). Two detectors were used, one detector having a temperature range of $26 \cdot 9-32 \cdot 4 \mathrm{C}$ and the other $26 \cdot 2-31 \cdot 3 \mathrm{C}$.

The examining membrane was applied to the trochanteric area to be examined and a permanent record of the stabilised picture was obtained with a purposemade Polaroid camera. Both the right and left trochanteric areas were examined under the same conditions with the same temperature range detector.

\section{References}

Guttmann L 1973 Spinal Cord Injuries, Comprehensive Management and Research. Blackwell Scientific Publications, p. 486.

JENkins DWR, Forster IW, MCKiBBin B, et al. 1977. Induction of tendon and ligament formation by carbon implants. Fournal of Bone and foint Surgery 59B:53-57.

Kosiak M 1959 Aetiology and pathology of ischaemic ulcers. Archives of Physical Medicine and Rehabilitation 40:62-69.

Minns RJ, SutTon RA 1982 Pressures under the ischium detected by a pedobarograph. Engineering in Medicine II: $11-16$.

Pochaczevsky R, Wexler CE, Meyers PH, et al. 1982 Liquid crystal thermography of the spine and extremities. Fournal of Neurosurgery 56:386-395.

Sutton RA, Bentley M, CAStree B, et al. 1982 Review of social situation of paraplegic and tetraplegic patients rehabilitated in the Hexham Spinal Injury Unit in the north of England over the past four years. Paraplegia 20:71-79.

TAyton K, Phillips G, Rallis Z 1982 Long term effects of carbon fibre on soft tissues. Fournal of Bone and foint Surgery 64B:112-114. 